\title{
ALGORITHM COMPARISON FOR REAL TIME KNOCK DETECTION
}

\author{
Stephan Ker, Frédéric Bonnardot, Laurent Duval
}

\author{
IFP \\ Technology, Computer Science and Applied Mathematics Division \\ 1 et 4 , avenue de Bois Préau \\ 92852 Rueil Malmaison Cedex - FRANCE \\ E-mail: \{stephan.ker, frederic.bonnardot, laurent.duval\}@ifp.fr
}

\begin{abstract}
This paper addresses the implementation and comparison of algorithms for real-time knock detection. Knock is an unwanted abnormal combustion process that may damage engines and limit their efficiency. For series vehicles, knock detection is generally obtained from knock sensors that capture other noise sources, thus requiring robust algorithms. In order to estimate the performance of time-frequency and Kalman filter based algorithms, a knock signal model is proposed and the algorithms are tested under various noise conditions. Experiments on modelled and real signals show the superiority of the recently developed S-method with respect to extended Kalman filtering.
\end{abstract}

Index Terms- Knock, Real time systems, TimeFrequency Analysis, Kalman filtering, Wigner distributions

\section{INTRODUCTION}

The knocking phenomenon in spark-ignition (SI) engines is a rapid burning of air-fuel mixture [1]. It appears like a fast pressure rise, which excites the chamber resonances and turns into oscillations that vibrate the entire engine. Heavy knocking conditions may damage the engine, and should therefore be avoided. However, knock often occurs near optimal combustion conditions. Due to higher requirements on fuel consumption, exhaust-gas limitations and engine knock, SI engines should nowadays work closer to the knocking limit. Its detection may involve in-cylinder pressure sensors. The latters provide precise information but they are relatively expensive and fragile for a series vehicle. Knock detection is usually performed with low-cost knock sensors mounted on the engine. As a consequence, the recorded signal contains external signals from other engine components such as valve openings and closings, piston slaps and additional noises with energy increasing with load and speed.

Therefore, knock detection algorithms should be designed with a particular attention paid to noise robustness. Moreover, for faster diagnosis, computation should be performed cycle-to-cycle. Standard algorithms use Fast Fourier transforms or integrated band-pass filtering, which are known to be noise sensitive, especially when precise knock parameters are looked for.

In this paper, we address the comparison between two types of robust real time algorithms for knock detection: timefrequency [2] or Kalman based [3]. Both are appropriate for envelope detection on narrow bandwidth signals. In Section 2 , we propose a simple yet representative model for the knock signal. It allows us to assess algorithms' noise robustness. Algorithms used for knock detection, namely the S-method and the expected Kalman filter approach, are briefly detailed in Section 3, with an emphasis on complexity and comments on the state space formalism. Section 4 deals with the simulation procedure, consisting in testing the algorithm sensitivity to increasing noise levels on the modelled knock signal. Performance is evaluated though simulations of multiple noise realizations. Then, experiments are performed on real world knock signals in Section 5, followed by final comments.

\section{THE KNOCK SIGNAL MODEL}

Several vibration signals have been acquired on a four inline cylinder direct injection engine with different speeds and loads and with a variation of the spark advance in order to meet the knock condition. The difference between non knocking and knocking condition can be identified by the apparition of a transient event with a narrow frequency bandwidth. A careful analysis of the vibration signal Wigner-Ville distributions shows that the knock related component may be characterized by a time-varying amplitude modulation and a nearly constant frequency content. The knock related vibration component may be modelled by a damped sinusoid, which can be expressed as:

$$
y(\alpha)=A_{0} \alpha e^{-d \alpha^{2}} \cos (c \alpha+\phi) .
$$

Where,

- $A_{0} \alpha e^{-d \alpha^{2}}$ represents the signal amplitude modulation, 
- the phase of the sinusoid is associated to the term $c \alpha+\phi$.

The damped sinusoid begins at $\alpha=0$ and is located within the normalized angle period $0 \leq \alpha \leq 1.5$.

In order to model the observed knocking signals, the parameters of the model introduced in (1) are $A_{0}=240, d=2$, $c=34 \pi$, and, $\phi=0$. This signal defined by these parameters is presented in Fig. 1.

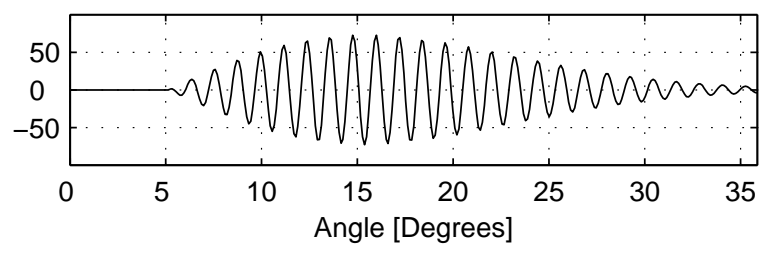

Fig. 1. Simulated knock signal.

\section{THE KNOCK SIGNAL DETECTION}

\subsection{Time-Frequency approach}

\subsubsection{S-method}

A first approach based on time-frequency analysis has been considered in order to detect the knock phenomenon in real-time. Classical time-frequency representations such as Wigner-Ville or Choi-Williams distributions are well suited to signal analysis for off-line diagnosis. Nonetheless, the computation time and the large number of operations required to perform such transforms often exceed on-board processor capabilities. An efficient way to obtain a time-frequency analysis in real-time applications has been introduced in [4] referred to as the $\mathrm{S}$-method. The $\mathrm{S}$-method principle is now shortly reviewed, a complete description of this method can be found in [5] and a VLSI implementation in [6]. The pseudo-Wigner distribution of a signal $x$ may be expressed as a function of the short-time Fourier transform (STFT) of the signal $x$ with the following expression:

$$
\begin{array}{r}
\mathrm{WD}_{x x}(t, \omega)=\frac{1}{\pi} \int_{-\infty}^{\infty} \operatorname{STFT}_{x}(t, \omega+\theta) \cdots \\
\cdots \times \operatorname{STFT}_{x}^{*}(t, \omega-\theta) d \theta .
\end{array}
$$

This relation leads to the S-method definition:

$$
\begin{array}{r}
\operatorname{SM}(t, \omega)=\frac{1}{\pi} \int_{-\infty}^{\infty} P(\theta) \operatorname{STFT}_{x}(t, \omega+\theta) \cdots \\
\cdots \times \operatorname{STFT}_{x}^{*}(t, \omega-\theta) d \theta
\end{array}
$$

where $P(\theta)$ is a finite frequency-domain window. Thus, the $S$-method estimates the Wigner distribution of the signal components separately avoiding cross-terms.

\subsubsection{S-method computed with SDFT}

Our approach is based on the S-method to analyze vibration signals in a narrow bandwidth where the knock phenomenon appears. This strategy requires the observation of only a small number of frequency components. As a consequence, instead of using the short-time Fourier transform in the computation of the S-method, the sliding discrete Fourier transform (SDFT) has been considered [7]. This transform performs the spectral time variation of a few frequency bins. This algorithm uses the circular property of the DFT in order to compute the spectral component $S$ for a frequency bin $k$ and for each sample $n$ with the following difference equation:

$$
S_{k}(n)=S_{k}(n-1) e^{j 2 \pi k / N}-x(n-N)+x(n),
$$

where $N$ is the number of samples of the considered time sequence. A discrete form of Equation (2), where $P(\theta)$ is a rectangular window with a constant width $L$, can be expressed as:

$$
\begin{aligned}
& \operatorname{SM}(n, k)=\frac{2}{N \pi}\left(\left|S_{k}(n)\right|^{2} \cdots\right. \\
&\left.\cdots+2 \sum_{i=1}^{L} \operatorname{Real}\left[S_{k+i}(n) S_{k-i}^{*}(n)\right]\right) .
\end{aligned}
$$

\subsection{Kalman filter approach}

\subsubsection{Simplified model}

For comparison purpose, a Schmidt's Extended Kalman Filter (EKF) based on Kalman filter [8] was designed to extract the knock signal. The knock model (1) is simplified into an amplitude-modulated cosine:

$$
z(n)=\lambda(n) \cos [\theta(n)]+w(n)
$$

where:

- $z(n)$ represents the $n^{t h}$ sample of the knocking signal,

- $\lambda(n)$ is slowly varying amplitude modulation relative to the carrier,

- $\theta(n)$ is the phase associated to the knocking phenomenon,

- $w(n)$ is a white observation noise which is assumed to be Gaussian.

Although the signal does not only contain knock phenomenon, the detection is made by looking at the frequency tracked by $\theta(n)$. To remain coherent with the S-method approach, the carrier frequency is considered constant (5). In fact, it is shown in the literature that it is not always the case [5]. Therefore, it should be pointed out that it is possible to model polynomial phases in the state equation [9]: 


$$
\begin{aligned}
\theta(n+1) & =\theta(n)+\Delta_{\theta}(n), \\
\Delta_{\theta}(n+1) & =\Delta_{\theta}(n)+v_{2}(n) .
\end{aligned}
$$

Since the amplitude modulation $\lambda(n)$ is slowly varying relatively to the carrier, it is locally approximated by a constant (6). In order to let the Kalman filter adjust this parameter, the slow variation is modeled by an additive Gaussian noise $v_{3}(n)$ :

$$
\lambda(n+1)=\lambda(n)+v_{3}(n) .
$$

Such a model is also known as the random walk. The noise variance should be tuned carefully: a high value results in observation noise fitting, a low one in a low reactivity.

\subsubsection{State space equation}

This model can be written as a state space model with a nonlinear observation equation and a linear state equation:

$$
\begin{aligned}
\mathbf{x}(\mathbf{n}+\mathbf{1}) & =\mathbf{F} \mathbf{x}(\mathbf{n})+\mathbf{v}(\mathbf{n}) \\
z(n) & =\mathbf{h}[\mathbf{x}(\mathbf{n})]+\mathbf{w}(\mathbf{n})
\end{aligned}
$$

where:

$$
\text { - } \mathbf{x}(\mathbf{n})=\left[\begin{array}{lll}
\theta(\mathbf{n}) & \boldsymbol{\Delta}_{\theta}(\mathbf{n}) & \lambda(\mathbf{n})
\end{array}\right]^{\mathbf{T}} \text { is the state vector, }
$$$$
\text { - } \mathbf{F}=\left[\begin{array}{lll}
1 & 1 & 0 \\
0 & 1 & 0 \\
0 & 0 & 1
\end{array}\right], \mathbf{v}(\mathbf{n})=\left[\begin{array}{c}
0 \\
v_{2}(n) \\
v_{3}(n)
\end{array}\right]
$$

- $z(n)$ is the observation.

The nonlinear observation function $\mathbf{h}[\mathbf{x}(\mathbf{n})]$ is:

$$
\mathbf{h}[\mathbf{x}(\mathbf{n})]=\lambda(\mathbf{n}) \cos [\theta(\mathbf{n})] .
$$

\subsubsection{Noise covariance tuning}

Since it is an order 2 method, the EKF algorithm requires a noise covariance knowledge.

The observation noise covariance associated to $w(n)$ is estimated by using a knock-free part of the signal. To setup the amplitude modulation variance $\sigma_{v_{3}}^{2}$ associated to $v_{3}(n)$, we allow a deviation of 10 percent of the maximum amplitude of a knock signal. Therefore, $\sigma_{v_{3}}^{2}=0.01 \max [z(n)]^{2}$.

A similar method is used to tune the phase increment variance $\sigma_{v_{2}}^{2}=0.01 \hat{c}^{2}$, where $\hat{c}$ is the approximative knock carrier frequency.

\subsubsection{Amplitude modulation smoothing}

Since the estimated amplitude modulation term $\lambda(n)$ is slowly varying, a smoothed version $\lambda_{s}(n)$ is used in order to reduce noise. The smoothing is done by a Savitzky-Golay filter [10].

EKF equations are well known [3], they will therefore not be developed here. It should be noticed that EKF uses a linearization (i.e. an order 1 Taylor development). Therefore, it is not as efficient as the Kalman filter (an Unscented Kalman Filter have been developed in [11] to provide a better solution than the "light" approximation of EKF).

\section{SIMULATION RESULTS}

To test the performance of the methods, the sampled knock signal $y(n)$ presented in Eq. (1) is embedded in a centered additive Gaussian white noise $w(n)$ of variance $\sigma_{w}^{2}$ :

$$
z(n)=y(n)+w(n) .
$$

The benchmark consists in estimating the amplitude modulation for a set of simulated signal with an increasing noise variance. The left part of Fig. 2 represents one realization of the noisy knock signal for three different SNRs: $12 d B$, $4 d B,-3 d B$. One can observe in the right part of Fig. 2 the good fitting of the S-method even in the worst case. The EKF seems to be more sensitive to the presence of noise.

To address more precisely the fitting quality, the mean square error between estimated and real amplitude modulations has been computed for several SNRs varying from $-3 \mathrm{~dB}$ to $12 \mathrm{~dB}$. For each SNR, 100 realizations have been drawn. The results are shown in Fig. 3. The standard deviation is also indicated by bars.

This statistical study confirms the good behavior of the Smethod and the weakness in strong noise environment of our EKF.
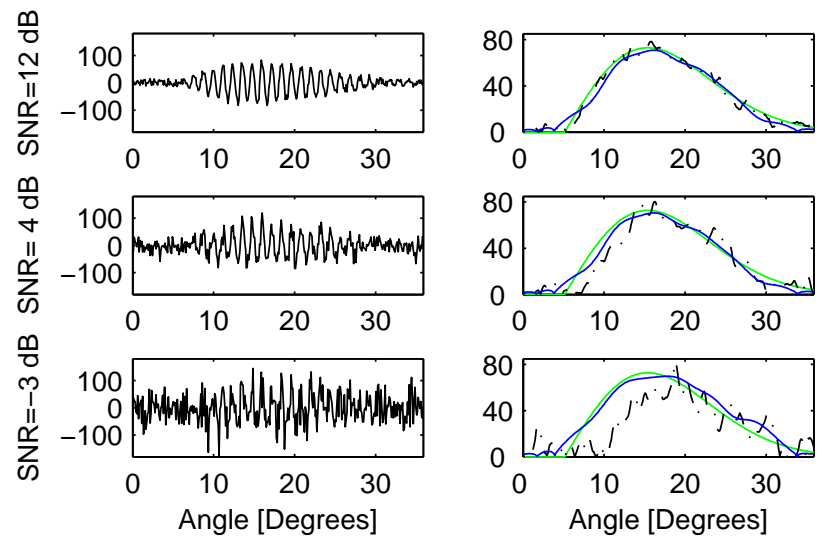

Fig. 2. Synthetic signals on the left. Exact (blue) and estimated amplitude modulation form the S-method (green) and EKF (dash). 


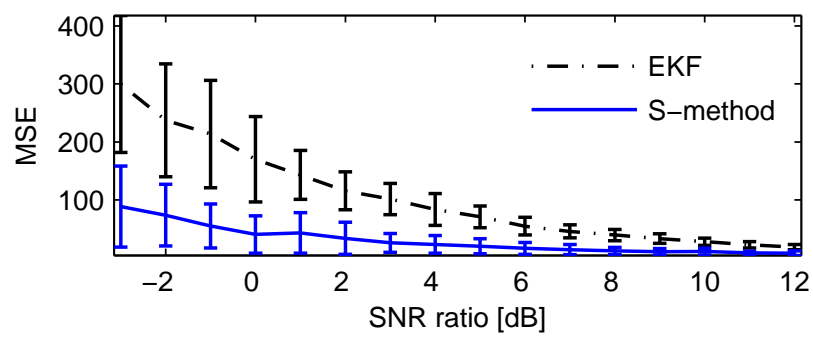

Fig. 3. Performance evaluation (the bar indicates the standard deviation).
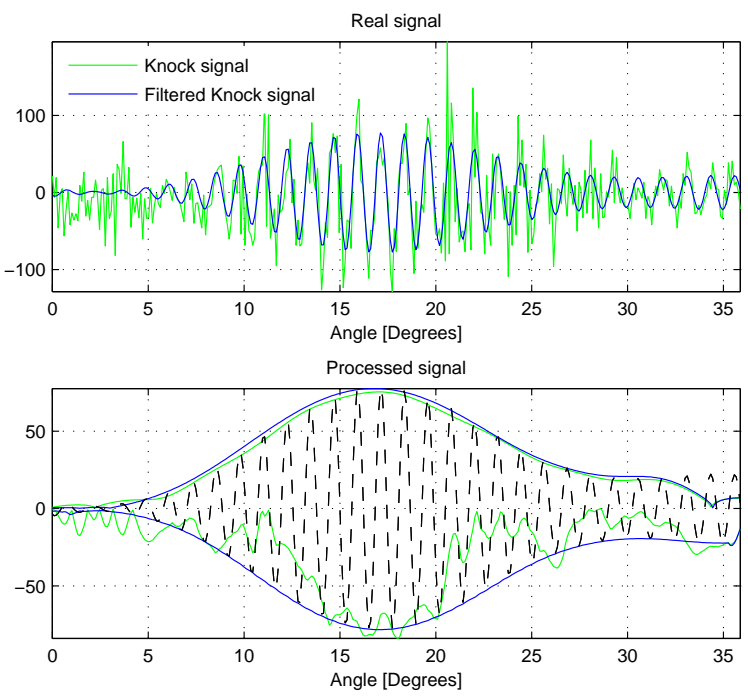

Fig. 4. Application on a real signal, with raw (green) and blue (filtered) envelope estimation.

\section{APPLICATION ON REAL SIGNAL}

This application is performed on an spark injection engine running at a speed of $1500 \mathrm{rpm}$. A real vibration signal related to the occurrence of the knock phenomenon is processed by the two approaches with and without a band pass filtering between 6 and $9 \mathrm{kHz}$. From this experiment presented in Fig. 4 , on the one hand the S-method (top envelopes) performs equaly well with or without filtering; on the other hand, the EKF (bottom envelopes) also provides a quite good estimation for the filtered signal only. A sharp study of the signal reveals that the carrier frequency is not perfectly constant (slight linear frequency modulation). It explains that EKF provides a better fitting than S-method in low SNR and linear frequency modulation case.

\section{CONCLUSIONS}

This paper presents a comparative study between two algorithms suited for real-time knock detection, the S-method based on a time-frequency approach and an extended Kalman filter. The results demonstrate the superiority of the S-method especially in presence of strong Gaussian noise. The good envelope estimation associated to the low computation requirements of the S-method allow a better characterization of the knock phenomenon than standard binary classification in "knocking" or "non knocking" conditions. Optimal SI engine performance may be reached by using close-loop control algorithms with knock detection based on the S-method.

\section{REFERENCES}

[1] M. Urlaub and J. F. Böhme, "Evaluation of knock begin in spark-ignition engines by least-squares," in Proc. Int. Conf. on Acoust., Speech and Sig. Proc., Philadelphia, USA, Mar. 2005.

[2] P. Flandrin, Time-frequency and time-scale analysis, Academic Press, San Diego, USA, 1998.

[3] C. K. Chui and G. Chen, Kalman Filtering with RealTime Applications, Springer-Verlag, New-York, USA, 3rd edition, 1998.

[4] LJ. Stanković, "A method for time-frequency analysis," IEEE Trans. on Signal Proc., vol. 42, no. 1, pp. 225229, 1994.

[5] LJ. Stanković and J. Böhme, "Time-frequency analysis of multiple resonances in combustion engine signals," Signal Processing, vol. 79, no. 1, pp. 15-28, 1999.

[6] S. Stanković, L. J. Stanković, V. Ivanović, and R. Stojanović, "An architecture for the VLSI design of systems for time-frequency analysis and time-varying filtering," Annales des Telecommunications, vol. 57, no. 9/10, pp. 974-995, Sept./Oct. 2002.

[7] E. Jacobsen and R. Lyons, "The sliding DFT," Signal Processing Magazine, vol. 20, no. 2, pp. 74-80, 2003.

[8] R. E. Kalman, "A new approach to linear filtering and prediction problems," Transactions of the ASMEJournal of Basic Engineering, vol. 82, Series D, pp. 35$45,1960$.

[9] M. Djeddi and M. Benidir, "A robust estimator for polynomial phase signals in non gaussian using parallel unscented Kalman filters," in Proc. Eur. Sig. and Image Proc. Conference, Florence, Italy, Sept. 2006.

[10] A. Savitzky and M. J. E. Golay, "Smoothing and differentiation of data by simplified least squares procedures," Analytical Chemistry, vol. 36, pp. 1627-1639, 1964.

[11] S. J. Julier and J. K. Uhlmann, "A new extention of the Kalman filter to nonlinear systems," in Proc. of AeroSense: The 11th Int. Symp. on Aerospace/Defense Sensing, Simulation and Controls, 1997. 